\title{
Drug Repurposing and the Medicinal Chemist
}

\author{
Jeffrey Aubé* \\ Department of Medicinal Chemistry, University of Kansas, Delbert M. Shankel Structural Biology Center, 2034 Becker Drive, \\ Lawrence, Kansas 66047-3761, United States
}

ABSTRACT: Drug repurposing is an approach to finding new uses for older drugs and has been gaining popularity in recent years. The role of traditional medicinal chemistry in the context of these efforts is considered.

$\mathrm{E}$ very practicing medicinal chemist labors under an assumption that is almost never stated out loud: not all potentially useful drugs for human use have yet been found. It certainly seems like a reasonable viewpoint, given the number of medical conditions either for which there is no pharmacotherapy available or for which existing treatments leave much to be desired. However, another way of addressing this need has gained steam in recent years. "Drug repurposing" is the practice of looking for new clinical uses of existing drugs, which contrasts sharply from de novo drug discovery approaches to therapeutics. The purpose of this essay will be to consider this approach, contrast it to traditional medicinal chemistry, and consider how the two approaches could positively complement each other.

The primary concern of all who engage in applied biomedical research should be helping patients in the absence of disciplinary bias. For the medicinal chemist, this means that the goal is to identify the best drug regardless of provenance or commercial concerns (including the recognition that drug therapy itself is not always the best course of action). Of course, pragmatic compromises must usually be considered, whether scientific or economic in nature. "Best" has a temporal connotation as well: the "best" drug today can change as new agents are introduced or as new information is obtained in and beyond clinical trials. For example, there are numerous conditions for which patients would gladly accept an imperfect cure, especially if one does not presently exist. In such cases, the lives of those who suffer improve to some degree, immediately. The state-of-the-art therapies in such areas as Alzheimer's or Parkinson's disease, as well as many types of cancer, can very much be viewed in this way. Attaining such a status quo does not mean that all research toward better treatments will stop. To the contrary, this is one area where the self-correcting nature of science flowers best, as scientists and clinicians work together to build a better tomorrow on top of yesterday's achievements.

One of the hardest parts of a de novo drug discovery campaign is starting out, in part due to the challenges of selecting and establishing initial structure-activity relationships on a given chemical series to explore. The short-term assessment of chemical series may be relatively easy to uncover through the selection of appropriate assays and biological models selection, but selecting the right series-i.e., one able to surmount all of the hurdles between discovery chemistry and the clinic-is much harder for the nonclairvoyant. Conventional wisdom has deemed most of the innovations meant to increase passage from early- to late-stage drug discovery wanting, especially the coupling of combinatorial chemistry with highthroughput screening. ${ }^{1}$ Although the idea that combinatorial chemistry or any other individual approach has failed is debatable, one thing that everyone can agree on is that it is harder than ever to develop a new drug and that these challenges have negatively impacted the global pharmaceutical enterprise.

Enter drug repurposing.

Generically, drug repurposing is a collection of approaches that collectively seek to adapt the current pharmacopeia for new uses. ${ }^{2-4}$ Included in the complicated taxonomy that is being developed for such approaches ${ }^{5}$ is "drug rescue", in which promising compounds that have been developed for one indication but have failed to reach the clinic are redirected toward another. For the purposes of this discussion, I will not attempt to differentiate between different flavors of drug repurposing but consider the concept in broad strokes.

The proponents of drug repurposing cite numerous scientific advantages of the idea. To my mind, foremost among these is related to a prime challenge in moving a molecule discovered by target-centric biology forward, namely establishing the validity of a new biological target in the treatment of disease. In this view, a considerable amount of time may be saved, as clinical trials would have been facilitated by the fact that the fictional repurposed candidate would have already been approved for use in humans. Other advantages attributed to repurposed drugs accrue from the fact that so much is known about them relative to newly synthesized molecules. As a class, they have at least tolerable safety and pharmacokinetic profiles, or they would not have been approved in the first place; minimally, one knows what one is dealing with (although it must be noted that, for drug rescue programs, one also knows that one is dealing with drug candidates that have, in fact, failed to reach the clinic). There are no hidden issues with respect to manufacturing or stability issues, and indeed, many drugs are off patent and may provide relatively inexpensive solutions for new problems. And they are available. Pragmatically, one can dovetail a repurposing effort with screening by the modest expedient of replacing a traditional screening library, which often contains hundreds of thousands of compounds, with a much smaller library of approved drug candidates. Such a

Published: May 24, 2012 
library is a key component of one important approach to drug repurposing/rescue being carried out under the banner of the newly formed National Center for Advancing Translational Science. ${ }^{6-8}$ Careful combinatorialization of the screening effort might uncover novel combinations of agents that are superior to single compounds, an approach that would be harder to apply to larger numbers of relatively unknown compound streams that would still require optimization (as might the repurposed drugs as well, but more about that shortly).

Other, nonscience-based factors have been partly responsible for the uptick in drug repurposing efforts, especially in academic- or foundation-based drug discovery efforts, many of which do not have at their beck and call a team of highly skilled medicinal chemists. For universities and research institutes seeking to establish themselves as bona fide players in drug discovery, a significant milestone is entry of a compound into clinical trials. The attractiveness of the repurposing approach for that milestone is obvious, even if the validity of entry into clinical trials as the primary measure of success (as opposed to successful passage through clinical trials into the clinic) is subject for discussion. Moreover, when confronted with the recognized difficulties and crushing expense of bringing a molecule all the way from discovery/ design/optimization and into the clinic, the allure of a repurposing approach is understandable.

What does all of this say about the role of the medicinal chemist in the twenty-first century? Some are quick to point out the downsides of repurposing, ranging from the lack of understanding of how the molecules are working (i.e., when the repurposed drug arose from a phenotypic or alternative assay lacking resolution vis-à-vis target) to the challenges of formulating a workable business model for patenting and employing a treatment that someone already owns. However, to defend traditional drug discovery by pointing to these concerns would be a cop-out. If real cures are to be found through drug repurposing of any ilk, creative solutions to its problems will not be far behind. And we owe it to patients to provide help regardless from which scientific approach the help arises or who benefits. (Remember that stuff about "identifying the best drug regardless of provenance"? I meant it.)

It is always dangerous to make predictions and doubly so to do it in print, but here goes. I suspect that drug repurposing, from a strictly scientific perspective, will grow in popularity as its potential is demonstrated and successes are seen. But like combinatorial chemistry and nearly every other "new" technology or approach, it is likely to reach a point where limits become more and more clear. At this point, discovery tools tend to reach their appropriate equilibrium and become accepted, warts and all, for what they are. Unless, in the process, it becomes clear that every useful drug molecule has indeed already been discovered (which is so unlikely, given the vastness of chemical space and the diversity of both target- and nontarget-based challenges in negotiating the biological milieu), de novo and repurposing approaches to drug therapy discovery will coexist.

While giving drug repurposing its chance to succeed or fail on its own merits, I'd like to advocate for maintaining a strong pipeline of drugs discovered and developed through de novo medicinal chemistry. This is due to the unique ability of synthetic medicinal chemistry to provide and optimize novel chemical matter and my strong sense that the need for such compounds is not going to end anytime soon. Drug repurposing's or, especially, drug rescue's reliance on finding a molecule in just the right chemical spot to cross the goal line is analogous to scoring in American football via pass interception or fumble recovery near the goal line. It is great when it happens, but successful football teams need a diversified strategy that also includes the long game, as tough as it can be. To this point, a case can be made that the additional time needed to optimize a given agent through SAR may not be the overwhelming cost driver in current drug development when compared to the cost of clinical studies.

Moreover, far from feeling threatened by drug purposing as a competing strategy, the medicinal chemist should use this tool when it makes sense to do so. Two limiting conditions can be envisioned for a successful repurposing project. In one, the drug acts at the same single target but with different outcomes that depend on the physical site of biological action. Even if the same drug were to be useful in both contexts, one can easily envision different distribution or metabolism issues that would require additional structural tweaking of the compound. Taking advantage of such a situation does require that the medicinal chemist come ready to ply her or his trade in the service of manipulating pharmaceutic properties-which I would argue ought to be part of every discovery scientist's personal toolbox in any circumstance.

The opposite end of the spectrum leads to even more clearcut conclusions. If the "old" purpose of the drug and the "new" one have different biochemical targets, it is extremely unlikely that the repurposed drug has been preoptimized for the latter situation. In other words, there is no reason to suppose that a structure-activity relationship campaign carried out to optimize a compound for target A would be identical to that needed for compound $\mathrm{B}$. This leads to the familiar situation, described above, where the repurposed drug, even if first-inclass to the clinic, represents a tentative solution that would eventually be rendered obsolete by a subsequent drug that would be even better. Medicinal chemists should feel enabled to tackle such a "fast follow-on" approach to new chemical matter, but to do so, they will have to come to grips with the understanding that the best way forward may not allow them to have the satisfaction of having invented the whole scaffold from the project's inception (IP attorneys will have to deal with the business and legal aspects of the same realization as well). Some solace may be taken from the fact that, if the repurposed drug is a member of a privileged class of chemical matter, many of the synthetic analogues needed may well already exist in the physical universe and be available at relatively modest expense.

As long as the field has existed, medicinal chemistry has sought to incorporate new tools and approaches to accomplish its mission of providing society with new and better drugs. Drug resourcing need not deter us from this path, even if it means that the mission statement will sometimes be edited to read "providing society with better drugs that are not necessarily so new". So long as the field of medicinal chemistry continues to demonstrate its worth by providing novel solutions to important problems, and so long as these efforts are supported by the business and academic research communities, we will earn our place in the global biomedical research community.

\section{AUTHOR INFORMATION}

\section{Corresponding Author}

*E-mail: jaube@ku.edu. 
Notes

The author declares no competing financial interest.

\section{ACKNOWLEDGMENTS}

The author thanks Professor Thomas Prisinzano for helpful comments.

\section{REFERENCES}

(1) For an appraisal of the combinatorial chemistry controversy from a contemporary perspective, see: Kennedy, J. P.; Williams, L.; Bridges, T. M.; Daniels, R. N.; Weaver, D.; Lindsley, C. W. Application of Combinatorial Chemistry Science on Modern Drug Discovery. J. Comb. Chem. 2008, 10, 345-354.

(2) Ashburn, T. T.; Thor, K. B. Drug Repositioning: Identifying and Developing New Uses for Existing Drugs. Nat. Rev. Drug Discov. 2004, $3,673-683$.

(3) O’Connor, K. A.; Roth, B. L. Finding New Tricks for Old Drugs: An Efficient Route for Public-Sector Drug Discovery. Nat. Rev. Drug Discov. 2005, 4, 1005-1014.

(4) Oprea, T. I.; Bauman, J. E.; Bologa, C. G.; Buranda, T.; Chigaev, A.; Edwards, B. S.; Jarvik, J. W.; Gresham, H. D.; Haynes, M. K.; Hjelle, B.; Hromas, R.; Hudson, L.; Mackenzie, D. A.; Muller, C. Y.; Reed, J. C.; Simons, P. C.; Smagley, Y.; Strouse, J.; Surviladze, Z.; Thompson, T.; Ursu, O.; Waller, A.; Wandinger-Ness, A.; Winter, S. S.; Wu, Y.; Young, S. M.; Larson, R. S.; Willman, C.; Sklar, L. A. Drug Repurposing From an Academic Perspective. Drug Discov. Today: Ther. Strategies 2011, 8, 61-69.

(5) Doan, T. L.; Pollastri, M.; Walters, M. A.; Georg, G. I.; Macor, J., E. . The Future of Drug Repositioning: Old Drugs, New Opportunities. In Annu. Rep. Med. Chem.., Macor, J. E., Ed. Academic Press: Oxford, 2011; Vol. 46, pp 385-401.

(6) Huang, R.; Southall, N.; Wang, Y.; Yasgar, A.; Shinn, P.; Jadhav, A.; Nguyen, D.-T.; Austin, C. P. The NCGC Pharmaceutical Collection: A Comprehensive Resource of Clinically Approved Drugs Enabling Repurposing and Chemical Genomics. Science Translational Med. 2011, 3, 80ps16.

(7) Collins, F. S. Mining for therapeutic gold. Nat. Rev. Drug Discov. 2011, 10, 397-397.

(8) The Discovering New Therapeutic Uses for Existing Molecules initiative was announced by NIH on May 3, 2012: http://www.ncats. nih.gov/research/reengineering/rescue-repurpose/therapeutic-uses/ therapeutic-uses.html. 